\title{
Investigation of the Dispersion Behavior of Inertial Particles within Accelerated Domain
}

\author{
T. Zhao ${ }^{1,2 \dagger}$, Z. Wang ${ }^{1}$,M. Takei ${ }^{2}$, K. Liu $^{1}$ and Y. Cui ${ }^{1}$ \\ ${ }^{1}$ Faculty of Mechanical and Precision Instrument Engineering, Xi'an University of Technology, Xi'an 710048, China \\ ${ }^{2}$ Division of Artificial Systems Science, Graduate School of Engineering, Chiba University, Chiba 263-8522, Japan \\ †Corresponding Author Email: tongzhao@xaut.edu.cn
}

(Received April 23, 2013; accepted June 10, 2013)

\begin{abstract}
This report presents a study of the dynamics dispersion behaviors of inertial particle in solid-air two-phase flow within accelerated domains using both experimental and simulation approaches. In the simulation, a threedimensional model was proposed by means of the combined computational fluid dynamics and a discrete element method (CFD-DEM). The simulation model provides information regarding the particle distribution behaviors and the particle run-away rate from the calculation domain. In the experiment, particle image velocimetry (PIV) and laser tomography were used to measure the particle velocity and concentration distribution, respectively. The simulation results were than validated by the experimental measurement. And the influence mechanisms of acceleration on the particle flow behavior were discussed in detail. As results, acceleration of the calculation domain affects the particle motion and causes a relative dense particles distribution in the accelerated direction. The particle run-out rate under acceleration was initially the same, but subsequently lower than that of the condition without acceleration. This finding shows that the acceleration adversely affects the particle run-out rate.
\end{abstract}

Keywords: Solid-air two-phase flow, Acceleration field, Particle dispersion behaviors, Particle image velocimetry.

\section{NOMENCLATURE}

$A_{L} \quad$ absorbance when light pass through the object field

$d_{p} \quad$ diameter of the particle

$f_{a} \quad$ fluid drag force exerted on the particle

$f_{c} \quad$ contact force

$f(x, y)$ object field distribution in direction $\mathrm{L}$ (x or y)

$G \quad$ gravitational force

$k_{n} \quad$ spring constant

$I_{0} \quad$ intensity of the light before it enters

the sample or incident light intensity

I intensity of light at a specified wavelength

$\lambda$ that has passed through a sample

$m \quad$ mass of the particle

$N p \quad$ initial loading particle number

$N_{\text {out }}$ number of particles that leave from the outlet during the time interval $\Delta t$ $n$

$p$

$R_{\text {out }}$

$T$

$U$

$v$

$v_{p}$

$\omega$

$\delta V$

$\varepsilon_{g}$

$\mu$

$\mu_{e}$

$\rho_{p}$ number of particles inside the cell pressure of the fluid

percentage of particle run out from the outlet at elapsing time $t$ torque caused by the contact force and the moment of inertia of the particle. velocity vector of the fluid translational velocity particle velocity rotational velocity volume of the computational cell void fraction fluid viscosity eddy viscosity particle density

\section{INTRODUCTION}

Particles of finite size, which have a mass density that differs from that of the carrier fluid, have inertia. These so-called inertial particles do not evolve as simple point-like fluid tracers. This property leads to preferential concentration, clustering, and separation of particles as observed in numerous studies (Tirumkudulu 
et al. 1999; Shinbrot et al. 2001). The inertial dynamics of solid particles can have important implications in natural phenomena, e.g., the transport of pollutants and pathogenic spores in the atmosphere (Schmale et al. 2005) and rain initiation by droplet coalescence in warm clouds (Shaw 2003). Several theoretical and numerical studies have examined the dynamics of inertial particles in model flows, such as the clustering of inertial particles in a two-dimensional flow past a cylinder (Haller et al. 2008), and similar studies have considered inertial particle clustering in turbulent flows (Bec 2003).

In Japan, an increasing number of residents suffer every year from allergic rhinitis, which is typically triggered by small particles, such as pollen, within the atmosphere. One solution to this problem is to purify the air of these particles by adding filter systems in an enclosed space, such as a train cabin. To enhance the performance of the air purification system, it is important to clarify the dispersion behaviors of the particles in the cabin, especially during the acceleration process. However, relatively few studies about particle dispersion behaviors in an accelerated cabin can be found; thus, the efficiency enhancement of the air purification system in a high-speed train cabin is limited.

In this study, both numerical simulation and experimental validation approaches were used to investigate the dispersion behavior of particles of finite size and mass in an accelerated cabin. Commonly used mathematical models can be grouped into two categories: the continuum-continuum approach on a macroscopic level, e.g., the two-fluid method (Wang et al. 2008; Asgharzadeh et al. 2012), and the continuumdiscrete approach on a microscopic level, e.g., CFDDEM (the combined computational fluid dynamics and discrete element method) (Xu et al. 1997). Because of the complicated closure models of the two-fluid method describing the mass, momentum and energy transfer between multiple continuous phases, which are typically not universally valid (Michael et al. 2009), the CFD-DEM method is used in this study to track the trajectory and the state (e.g., velocity) of each individual particle.

When considering quantitative visualization for multiphase flow, the optical technique is a powerful diagnostic method for the concentration profiles of the multiphase flow. For measurement of particle concentration distribution, the orthogonal laser tomography technique has proven to be effective in the study of particle-fluid flow systems (Abdul Rahim et al. 1996, 1998; Green et al. 1998). For the velocity field, particle image velocimetry (PIV) is generally used to measure the local velocity in the flow field in a variety of fluid dynamic studies over different body configurations (Choi et al. 2002; Fan et al. 2009; Larsson et al. 2012). In most studies, these two technologies are used alone. In this study, the orthogonal laser tomography and PIV are combined to validate the simulation results provided by the CFDDEM. Specifically, the simulations are compared with the measurement results of particle concentration distribution from two optical fiber arrays and the results of the particle velocity distribution from the PIV system.

\section{Simulation Model}

\subsection{Solid Phase}

The numerical model used the continuum-discrete approach on a microscopic level for the physical interactions between particles, that is, an EulerianLagrangian model, was used to simulate the interested dynamics of particle distribution. In a conventional DEM, the movement of individual particles is evaluated by Newton's equation of motion, which includes the effects of gravitational force, contact force, and fluid force. The long-range interaction between particles, such as the Van der Waals force, electrostatic force and liquid bridge force, are neglected, and the direct contact force due to collisions is considered to be the most significant interface force. The translational and rotational motions of a particle at any time, $t$, are determined by the momentum balance, which is given as follows (Zhao et al. 2010):

$$
\begin{aligned}
& m d \mathbf{v} / d t=\mathbf{f}_{\mathbf{a}}+\mathbf{f}_{\mathbf{c}}+\mathbf{G} \\
& I d \boldsymbol{\omega} / d t=\mathbf{T}
\end{aligned}
$$

where $m$ is the mass of the particle; $\mathbf{v}$ and $\boldsymbol{\omega}$ are the translational and rotational velocities, respectively; $I$ is the moment of inertia; $\mathbf{f}_{a}$ is the force acting on a particle exerted by the surrounding fluid; $\mathbf{f}_{\mathbf{c}}$ is the contact force; $\mathbf{G}$ is the accelerating force including gravity; and $\mathbf{T}$ is the torque caused by the contact force and the moment of inertia of the particle.

Cundall and Strack (Cundall et al. 1979) proposed a preliminary model to formulate the particle-particle interaction. Based on their research, the contact force between two spherical particles can be modeled by the simple concepts of the spring, the dash-pot, and the friction slider. The contact force can be divided into a normal contact force and a tangential contact force (Tsuji et al. 1992,1993). Before the calculation of the contact force, the stiffness, the coefficient of viscous dissipation, and the friction coefficient, which can be obtained from the physical properties of the particles, must be determined first. In the current study, these parameters were determined by the method proposed in References (Tsuji et al. 1993,1993; Zhao et al. 2010). The contact force model is also used to simulate the interaction between the particle and the wall, while the mass of the wall is assumed to be infinitely large, and the velocity vectors are set to zero.

\subsection{Gas Phase}

The gas phase is treated as a continuous phase and is modeled in a manner similar to that used in the conventional two-fluid model. The governing equations are the conservation of mass and the conservation of momentum equations in terms of the local mean variables over a computational cell, which are given as follows (Zhao et al. 2010): 
$\partial \varepsilon / \partial t+\nabla \cdot(\boldsymbol{u u})=0$

$$
\begin{aligned}
& \partial\left(\rho_{g} \varepsilon_{g} \mathbf{u}\right) / \partial t+\nabla \cdot\left(\rho_{g} \varepsilon_{g} \mathbf{u u}\right) \\
& =-\varepsilon_{g} \nabla p+f_{a, i}+\varepsilon_{g}\left(\mu+\mu_{e}\right) \nabla^{2} \mathbf{u}
\end{aligned}
$$

where $\varepsilon_{g}$ is the void fraction; $\mathbf{u}$ is the velocity vector of the fluid; $p$ is the pressure of the fluid; $\mathbf{f}_{\mathbf{a}}$ is the fluid drag force exerted on the particle; $\mu$ is the fluid viscosity; $\mu_{e}$ is the eddy viscosity; $\delta V$ is the volume of the computational cell; and $n$ is the number of particles inside the cell. In this equation, the standard $k-\varepsilon$ model is used as the turbulence model. The fluid drag force can be calculated as follows (Tsuji et al. 1993):

$\mathbf{f}_{\mathrm{a}, \mathbf{i}}=\beta\left(\mathbf{v}_{\mathbf{p i}}-\mathbf{u}_{\mathbf{i}}\right) \delta V$

where $\mathbf{v}_{\mathbf{p}}$ is the particle velocity. The coefficient $\beta$ can be determined by Ergun's equation ( $\varepsilon \leq 0.8$ ) (Ergun 1952), or Wen and Yu's equation $(\varepsilon>0.8)$ (Wen et al. 1966). In this study, $\beta$ was calculated based on the equations summarized in Reference (Kawaguchi et al. 2000).

\subsection{Simulation conditions}

A salient feature of the suspensions in air is the presence of strong inhomogeneities in the spatial distribution of the particles. These particles are inhomogeneous in their distribution, shape and density. The dispersion behaviors of these particles will be directly affected by their size and density due to inertia. Because of their high deposition rate in the nose and throat of human beings, particles with a diameter in the range of 1 to $100 \mu \mathrm{m}$ are proven to be the main reason for allergic rhinitis. Therefore, only the motion of particles within this diameter range was studied. Furthermore, to maintain consistency between the simulation and the experiment, it is better to set the particle diameter in the simulation same as that in the experiment. However, the size of particle used in the experiment actually distribute in a wide range from severe to hundreds of micron. So the Sauter mean diameter of the particle used in the experiment, which is $d_{\mathrm{p}}=70 \mu \mathrm{m}$, was used as the particle diameter in the simulation.

Figure 1 shows the calculation domain of the simulation model, with a width of $80 \mathrm{~mm}$, a height of $80 \mathrm{~mm}$ and a length of $200 \mathrm{~mm}$. In the simulation, the air phase is injected from a square air inlet with dimensions of 24 $\mathrm{mm} \times 24 \mathrm{~mm}$ and ejected from two outlets with dimensions of $12 \mathrm{~mm} \times 12 \mathrm{~mm}$. Particles can only leave the calculation domain through the outlets. The simulation model was created and analyzed using the Rflow software provided by R-flow Corporation Ltd., Japan. As mentioned above, the calculation domain is divided into small calculation cells. The cell size for the calculation of gas motion is $4 \mathrm{~mm} \times 4 \mathrm{~mm} \times 4 \mathrm{~mm}$, and the total number of grid cells is 20,000 . To maintain the accuracy of the simulation and improve the computing speed the minimum calculation cell was chosen, which would not qualitatively affect the simulation result. Each cell consists of the gas phase contacting with the particles, and the void fraction of each cell can be defined by the number of particles existing in the cell. As is typical in many numerical calculations for flow

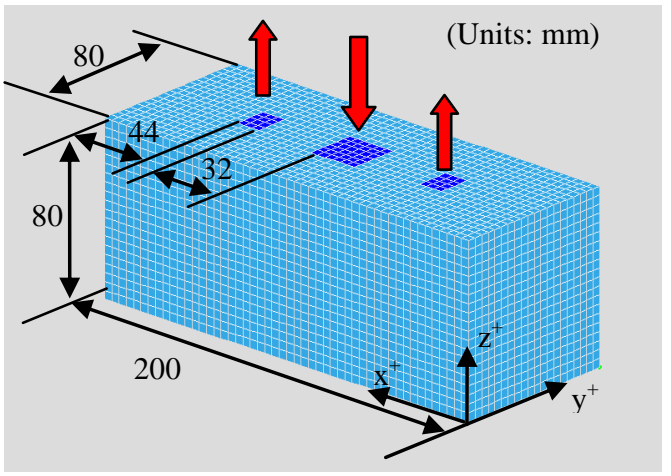

Fig. 1. Calculation domain of the simulation model

\begin{tabular}{|c|c|c|}
\hline \multicolumn{3}{|c|}{ Gas phase } \\
\hline Fluid & \multicolumn{2}{|c|}{ air } \\
\hline Density $\left(\mathrm{kg} / \mathrm{m}^{3}\right)$ & \multicolumn{2}{|c|}{1.205} \\
\hline Inject air velocity $(\mathrm{m} / \mathrm{s})$ & \multicolumn{2}{|c|}{1} \\
\hline Viscosity $(\mathrm{kg} / \mathrm{ms})$ & \multicolumn{2}{|c|}{$2.0 \times 10^{-5}$} \\
\hline Geometry $(\mathrm{m})$ & \multicolumn{2}{|c|}{0.20 (length) } \\
\hline & \multicolumn{2}{|c|}{0.08 (height) } \\
\hline & \multicolumn{2}{|c|}{0.08 (width) } \\
\hline \multirow[b]{2}{*}{$a_{x}$} & Case 1 & $a_{x}=-2 \mathrm{~m} / \mathrm{s}^{2}$ \\
\hline & Case 2 & $a_{x}=0 \mathrm{~m} / \mathrm{s}^{2}$ \\
\hline \multicolumn{3}{|c|}{ Particle phase } \\
\hline \multicolumn{2}{|c|}{ Particle shape: } & spherical \\
\hline \multicolumn{2}{|c|}{ Density $\left(\mathrm{kg} / \mathrm{m}^{3}\right)$} & 1,000 \\
\hline \multicolumn{2}{|c|}{ Spring constant $(\mathrm{N} / \mathrm{m})$} & 4 \\
\hline \multicolumn{2}{|c|}{ Friction coefficient } & 0.3 \\
\hline \multicolumn{2}{|c|}{ Particle diameter $(\mu \mathrm{m})$} & 70 \\
\hline \multicolumn{2}{|c|}{ Time step (s) } & $3.7 \times 10^{-6}$ \\
\hline \multicolumn{2}{|c|}{ Gravity acceleration $\left(\mathrm{m} / \mathrm{s}^{2}\right)$} & -9.8 \\
\hline \multicolumn{2}{|c|}{ Height of initial particle layer $(\mathrm{mm})$} & 2 \\
\hline
\end{tabular}

Table 1 Basic simulation parameters

fields, the differential equations of the gas phase were solved by the finite difference method. A well-known numerical method, the semi-implicit method for pressure-link equation (SIMPLE), was used. A no-slip condition is used for the air phase at the walls, and particles are allowed to collide with the wall. The calculation was carried out in a rectangular Cartesian coordinate system, as shown in figure 1 .

The initial particle layer at the bottom of the calculation domain has a height of $2 \mathrm{~mm}$ with an initial particle volume fraction of $15 \%$. Therefore, the initial total particle volume fraction in the calculation domain is around $0.4 \%$. Table 1 shows the simulation conditions and the basic parameter settings of the current study. In order to simulate the acceleration process of the high speed train cabin, the virtual acceleration $a_{x}$ was acted to provide the relative acceleration between particles and the calculation domain. The time step for the 
simulation is calculated by the equation proposed by Tsuji et al. (Tsuji et al. 1992) as follows:

$$
\Delta t=\frac{2}{5} \pi \sqrt{\frac{\pi\left(d_{p}\right)^{3} \rho_{p}}{6 k_{n}}}
$$

where $d_{p}$ is the diameter of the particle; $k_{n}$ is the spring constant; and $\rho_{p}$ is the particle density. From this equation, it is clear that the time step strongly depends on the spring constant. The actual spring constant will yield a small time step, which requires an extremely long computation time. In the current simulation, a smaller stiffness value was assumed to reduce the computation time. The time step for the simulation is $\Delta t=3.7 \times 10^{-6} \mathrm{~s}$ in the current simulation. Air is injected into the container uniformly to fluidize the particles.

\section{EXPERIMENTS}

\subsection{Experimental rig}

To evaluate the simulation results, a simple experiment was performed. The experimental procedure was designed to provide well-characterized particle dispersion behaviors during the acceleration process of the cabin. Two experimental instruments were used in this investigation. The first instrument was an optical tomography system in which laser measurements were conducted for the particle concentration field. Additionally, the PIV measurements were conducted at the same time for the particle velocity field. As shown in Fig. 2, the entire experimental system consists of the laser transmitter-receiver array (LV-H100, Keyence Corporation, Japan), charged-coupled device (CCD) camera, board screw, motor (MV-20, Keyence Corporation, Japan), signal conditioning and data acquisition device, plastic box and air supplier. The camera and laser arrays were fixed with the test cabin and accelerated together to obtain the particle velocity distribution behavior.

The experiment used the same geometry of the plastic box as the simulation, except that the air inlet/outlet was varied from a square to a circular shape due to the relative big cell size of the calculation domain. These changes of the geometry will not considerably influent the comparability of experiment and simulation results, for two reasons. First, although the geometry of the inlet and outlet is changed, the inject air flow rate is consistent. And the inject air flow rate is more dominate of the particle distribution in the investigate domain of present study. Secondly, compare with the size of the whole calculation domain, the inlet and outlet are really small and the geometry change can only affect the flow field in a limited area close to the inlet and outlet. The plastic box was connected with a board screw and accelerated via a motor. The accelerate distance was only $0.5 \mathrm{~m}$ because of the limited length of the board screw. The particulate solids used for this study were catalyst particles with a particle density of $1,000 \mathrm{~kg} / \mathrm{m}^{3}$ and a mean diameter of $70 \mu \mathrm{m}$.

\subsection{Laser measurement system}

The laser measurement system was developed based on the theory of laser tomography (Zheng et al. 2006).
Two sets of laser transmitter-receiver arrays are mounted around the measurement section to produce the orthogonal projections. Each set of the laser array consist of 8 transmitter-receiver pairs. Each transmitter corresponds with a specific receiver, and the resulting sensor is termed a transmitter-receiver pair. The optical axis of each transmitter is aligned with the optical axis of the opposite receiver.

Light from the laser source, which is generated with unfocused and collimating lenses, is conducted into the measurement cross section via a transmitter and is received at same time by a receiver opposite the laser source. Solid particles in the box travel through the optical sensitive field formed by the transmitterreceiver array; thus, attenuated light reaches the receiver. Light energy from the receiver is converted into an electrical signal by a current-to-voltage converter. The voltage is amplified and conditioned using A.C. coupled amplifiers to produce a response between the solid concentration and the voltage. The conditioned signal is sampled to produce a digital signal in a form suitable for further processing.

The projection of the object field intensity along a certain line can be expressed by the following equation (Bariter et al. 1996):

$\int_{L} f(x, y) d L=-\ln \left(\frac{I}{I_{0}}\right)=A_{L}$

where $f(x, y)$ is the object field distribution in direction $\mathrm{L}$ ( $\mathrm{x}$ or $\mathrm{y})$; $\mathrm{I}$ is the intensity of light at a specified wavelength $\lambda$ that has passed through a sample (transmitted light intensity); $\mathrm{I}_{0}$ is the intensity of the light before it enters the sample or incident light intensity (or power); and $A_{L}$ is the absorbance when light pass through the object field. This study assumes that the average concentration of the solids passing through a small cross-section of the box is a linear function of the number of particles intercepting the beams passing through this small cross-section. Furthermore, the number of particles passing through a beam clearly relate to the corresponding sensor output signal. Therefore, the particle distribution behaviors can be deduced from the measurements of $A_{L}$.

\subsection{PIV measurement system}

Typically, PIV employs tracer (or seeding) particles to estimate the velocity field of fluid. The tracer particles, which should be sufficiently small, are assumed to faithfully follow the flow dynamics. Motion of the seeding particles is used to calculate speed and direction (the velocity field) of the flow being studied. However, in the present study, PIV technique is use to validating the simulation result of particles dispersion behaviors provided by CFD-DEM. So the objective of PIV measurement is the inertial particles flow not the air flow, and only the velocity field of particle flow is necessary. So in the PIV experiment of this present study, there were no tracer particles in the air flow. Only the inertial particles are visible under illumination. The particle motion was recorded, and the velocity field of particle flow was calculated.

The PIV system consisted of a CCD camera, a data 
acquisition and process unit, and software running on a personal computer for system control, data

management, and data post-analysis. The

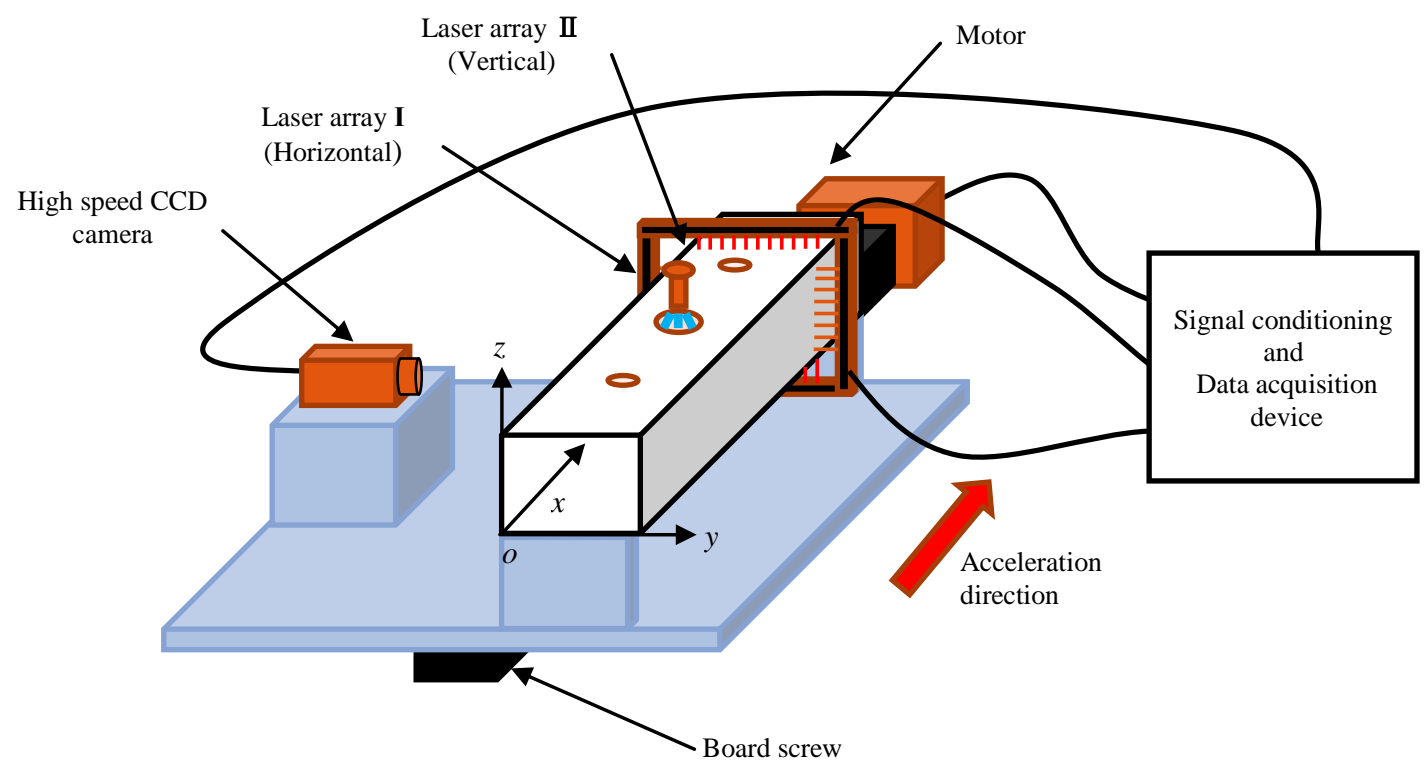

Fig. 2. Experimental setup

high-resolution $(1016 \times 1016$ pixel $)$ digital cameras and associated frame grabber boards have two modes of operation. The normal mode has a 30 frame per second (fps) framing rate. The 'triggered' mode enables the cameras to capture two consecutive frames with a very small time delay controlled by the user, while the system still maintains a capture rate of $50 \mathrm{fps}$. In the triggered mode, the effective framing rate for capturing small velocity scale flow is $15 \mathrm{~Hz}$ (using the first image of a triggered pair), while large velocity scales can be resolved with the triggered image pair. As frames are captured by the camera, they are transferred to a data acquisition unit for temporary storage. The device is capable of holding approximately 400 sequential images in RAM, resulting in $13.3 \mathrm{~s}$ of continuous run time before the frames must be written onto a hard disk. The illumination is supplied by light emitting diodes (LEDs), which face the cameras on the opposite side of the test volume. A screen diffuses the LED light, and filters are attached to the camera to remove reflected light. Since no laser sheet was supplied into the measurement domain, the camera actually captured particle motions in a well-lit 3D space. However, the particle volume fraction is very small (less than $0.4 \%$ ), and thus the image captured by the camera is more like a projection of the particle motions to a 2D-planar space. Moreover, PIV measurements involve instrument and algorithm chains with coupled uncertainty sources, rendering quantification of uncertainty far more complex. Ultimately in order to comprehensively quantify the uncertainty, all possible combinations of displacements, shears, rotations, particle diameters, and any other parameter used must be exhaustively tested which can make it computationally expensive. And many of the relevant parameters may not be easily obtained from a real experiment. As a second best, the effects of the uncertainties are averaged out by the random repetitive experiment (Raffel et al., 2007; Larsson et al., 2012). Therefore, the particle velocity distribution results obtained from the PIV measurement may miss some particles information and contain errors, but it still valuable for the qualitative validation of the CFD-DEM simulation.

The process unit changes the images to a raw vector map by a correlation method. There are two different modes available, namely, cross-correlation or autocorrelation. In auto-correlation mode the ensemble of particles is illuminated multiple times in a single recording. Therefore, in evaluating the auto-correlation two displacement correlation peaks (self-correlation peak and displacement peaks) arise, since the direction of the displacement is ambiguous. For small displacements this self-correlation peak may cover the displacement peaks. In cross-correlation mode each particle is illuminated only once in each sample. So, there is no self-correlation and since the order of the images is known there is no directional ambiguity. Compared to the auto-correlation mode, the crosscorrelation mode is relative simple and effective, and somewhat increase the spatial resolution due to the larger information contents of an image pair. Moreover, cross-correlation method is sensitive to the peak voltage in the sensor output and less sensitive to the noise and DC level. Therefore, the cross-correlation method is used in this study to analyze the raw data. After processing the image map to a raw vector map, the remaining analysis takes place on the computer. After applying different possible filtering options, there are primarily three analyzed data sets to obtain validated vector maps. To obtain information about the exact velocities of the particles in the image area, it is possible to list these data ordered by their spatial coordinates as numerical data.

\section{Results AND Discussion}

\subsection{Particle concentration distribution}


Table 2 shows the distribution images of the particle concentration distribution in the XZ plane while $y=0.04$

\section{Table 2 Particle concentration distribution images}

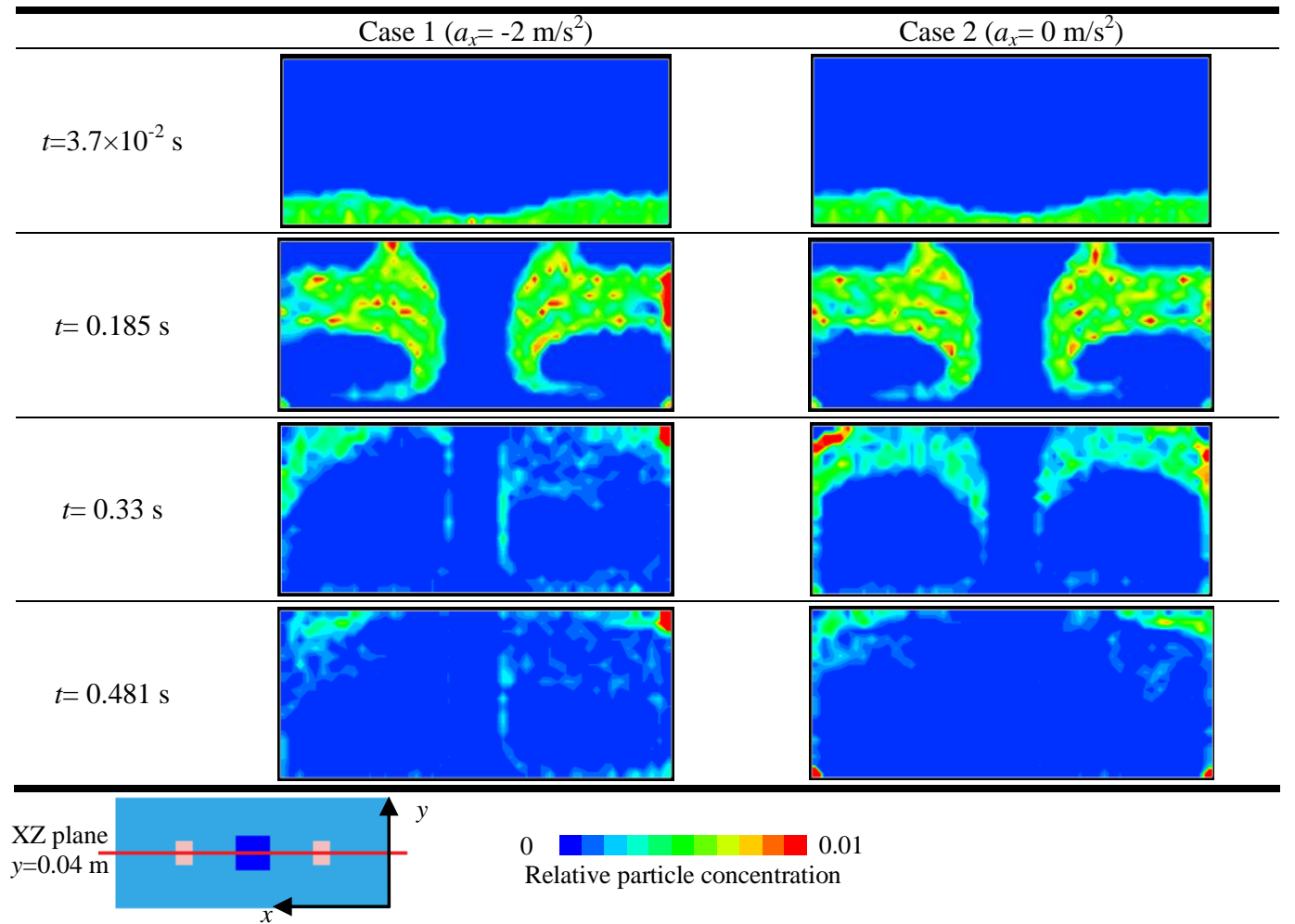

$\mathrm{m}$ from simulation for cases 1 and 2 . In these images, the red color indicates a high particle concentration, which is $1 \%$. As the particle concentration decreases, it turns blue, which indicates air, as shown by the color bar.

In table 2, it is clear that as air is injected through the inlet, particles formed two circulating flows separated by the air jet inlet in cases 1 and 2: one clockwise circulated at right and the other counter-clockwise circulated at left. The particle concentration decreases as time elapses. The reason for this phenomenon can be explained by noting that during the circulation, a portion of particles exited the calculation region from the outlet, while the remainder of the particles continued circulating. Additionally, it can be observed that as time increases, several high concentration areas are generated near the right wall due to the relative acceleration between particle and calculation domain in case 1 , whereas this phenomenon did not appear in case 2.

Table 3 shows the distribution images of the particle concentration distribution in the $\mathrm{YZ}$ plane at $x=0.128 \mathrm{~m}$ from simulation and the absorbance distributions obtained from the laser transmitter-receiver arrays for case 1 . From table 3 , it can be found that the absorbance distribution qualitatively reflect the particle distribution. As shown in Eq. (14), it is clear that the high absorbance value indicates that the integrated particle concentration in the $L$ direction is high, and the low absorbance indicates likewise. Therefore, the simulation results can be qualitatively assessed by the absorbance distributions.
From Table 3, it can be found that the simulation results agree qualitatively well with the experiment at the time $t=3.7 \times 10^{-2} \mathrm{~s}, 0.185 \mathrm{~s}$ and $0.33 \mathrm{~s}$, while the particle distribution is relatively dense. For example, at the sampling time $t=3.7 \times 10^{-2} \mathrm{~s}$, the absorbance distributions results show that the particles concentrate in the area of $z=0$ to $0.02 \mathrm{~m}$ and that the particles are uniformly distributed in the $y$ direction, except for a slight decrease in the center, which are consistent with the simulation results shown in the left. Similarly, the comparison between the simulation and experimental results at the sampling time $t=0.185 \mathrm{~s}$ and $0.33 \mathrm{~s}$ shows the same result. However, when the particle distribution becomes more dispersed $(\mathrm{t}=0.481 \mathrm{~s})$, little similarity can be found. This finding might be observed because only two orthogonal laser transmitter-receiver arrays are used in the experiment. In addition, when the particle concentration becomes dilute, the information from these two laser arrays is not sufficient to distinguish the particle distribution.

\subsection{Velocity distribution}

Figure 3 illustrates the simulation result of the distribution image of air velocity vector in the calculation domain for case 1 . In these figures, only the sectional velocity distribution in the $\mathrm{XZ}$ plane while $y=0.04 \mathrm{~m}$ is extracted, as shown by the annotation drawing. The red color vector indicates a high velocity of $2 \mathrm{~m} / \mathrm{s}$.

As shown in Figure 3, the air stream accelerates the stationary fluid and particles until it impinges onto the 
bottom. It is clear that there are two circulating airflow inlet and outlets. Additionally, it can be observed that routes generated due to the geometric position of the air

Table 3 Comparison between simulation and laser experiment (Case 1)

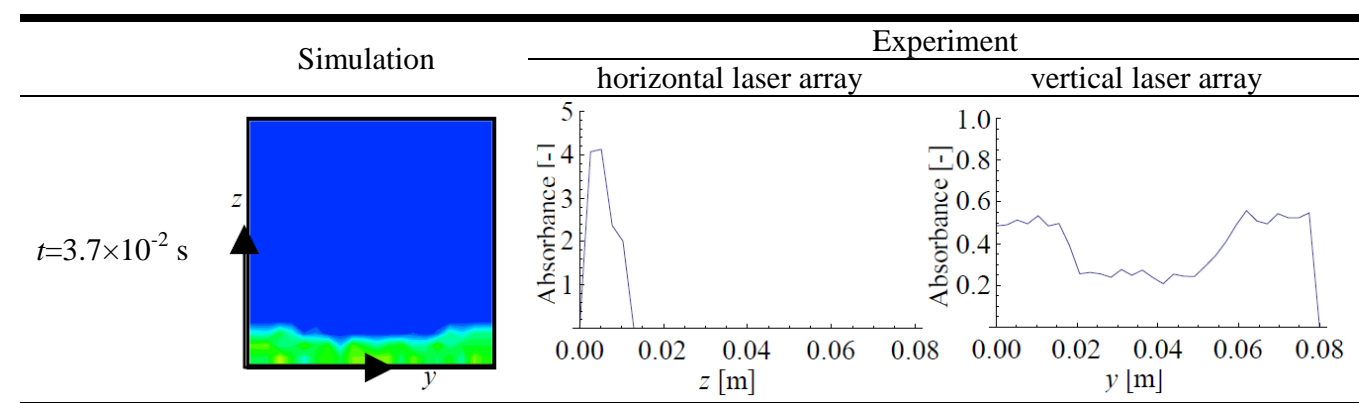

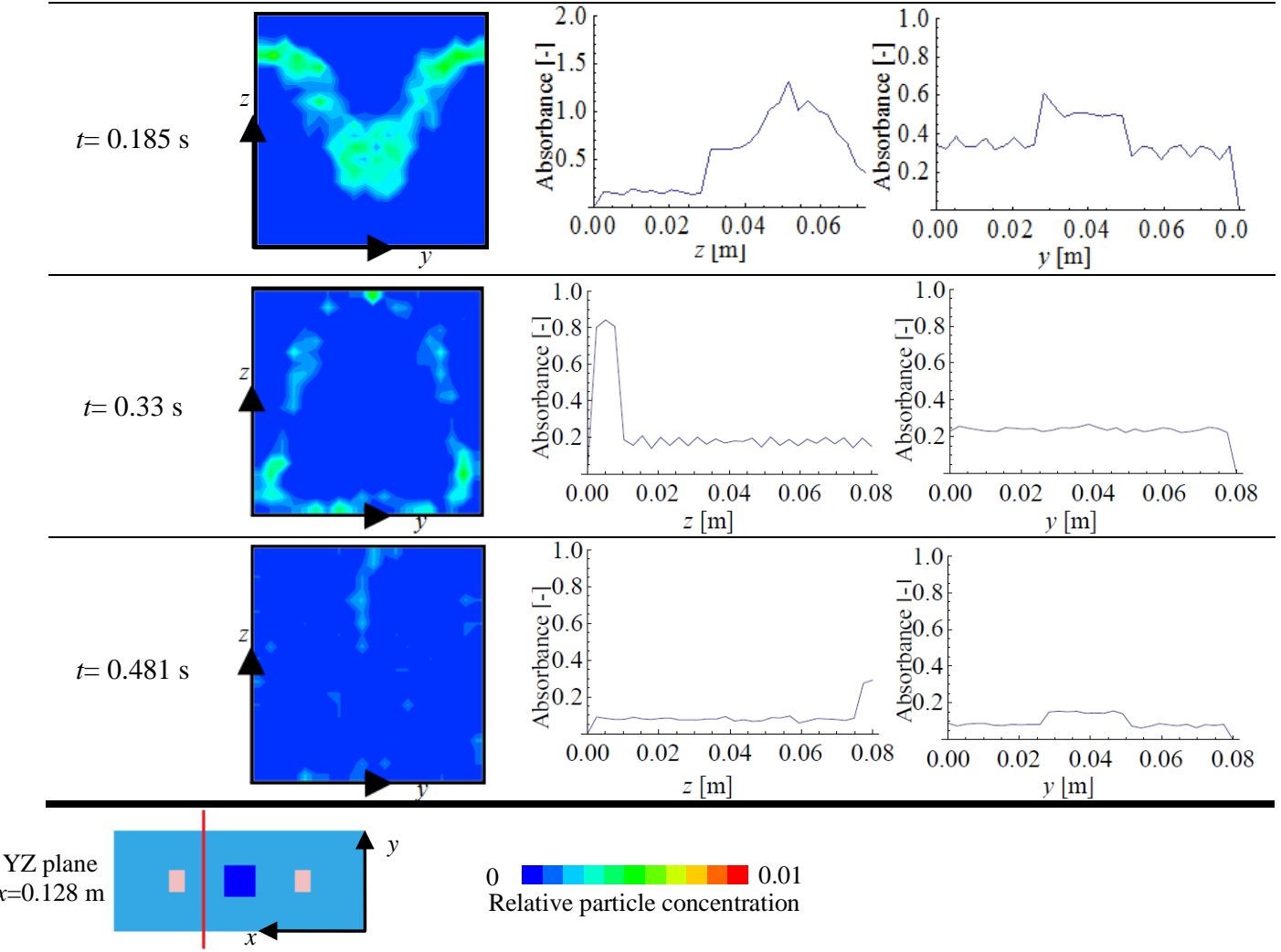

the air velocity distribution always remains constant and approximately stable at different times.

Figure 4 shows the distribution images of particle velocity at $y=0.04 \mathrm{~m}$ for case 1 . As shown in figure 4 , particles over the entire space experience the forces of the fluid, which carry them to the outlet areas. Therefore, it can be observed that the fluid drag force on the particles depend on the distance to the air supply. Increasing the distance reduces the forces on the particles. Then, it can be found that following the air flow, particles generate two circulating flow routes and leave the calculation domain when they cross through the outlet boundaries. Moreover, at time $t=0.185 \mathrm{~s}$ and $0.33 \mathrm{~s}$, the particle velocity vectors in the right circulating flow routes (or vortex) become more intensive than that in the left. Due to the acceleration influence, particles in the right circulating flow routes become difficult to run out of the calculation region.
PIV experiments were conducted to validate the phenomena shown above. Figure 5 shows the particle velocity distribution image at $t=0.1 \mathrm{~s}$ and $0.3 \mathrm{~s}$ provided by the PIV system. From figure 5(a), it can be found that the air flow from the inlet initiated the particle motion and performed vortex. The effect of wall friction is also observable in the vector length, which decreases if the particles are close to the wall. The stagnation point on the bottom under the middle air supply is also evident. The particle motion near the outlets is extremely slow, which is influenced by the boundary layer. Additionally, it is observed in Figure 5(b) that the particle velocity vector is more intensive in the right vortex, which shows the same behavior in Figure 4(c) and 4(d). This result indicates that the particles concentration in the circulating flow routes is adversely affected by the acceleration. However, the difference between the calculation and the experiment can also be observed in that a high particle velocity 
region always exists near the entrance of airflow in the experiment, whereas the existence of such a region disappears in the simulation. These differences can be considered to be due to the variations of the particle
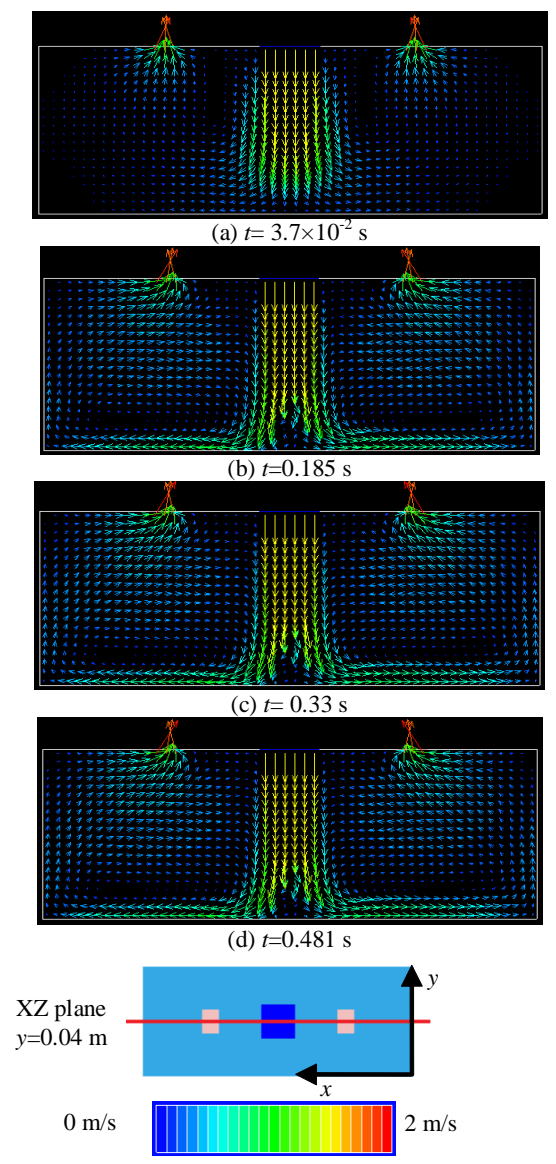

Fig. 3. Air velocity vectors in plane $\mathrm{XZ}$ from simulation (Case 1, $y=0.04 \mathrm{~m}$ )

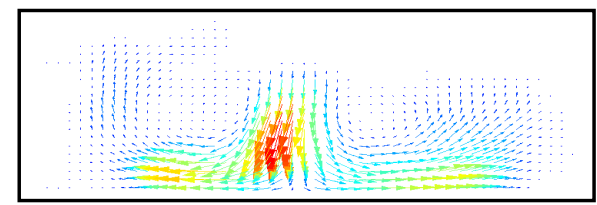

(a) $t=0.1 \mathrm{~s}$

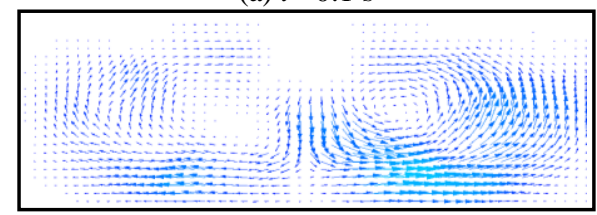

(b) $t=0.3 \mathrm{~s}$

Fig. 5. Particle velocity distributions by PIV (Case 1)

size distribution and the particle shape compared to the actual experimental condition. Therefore, future research should focus on improving the simulation model with multi-sized and multi-shaped particles to achieve a better qualitative agreement or even quantitative agreement
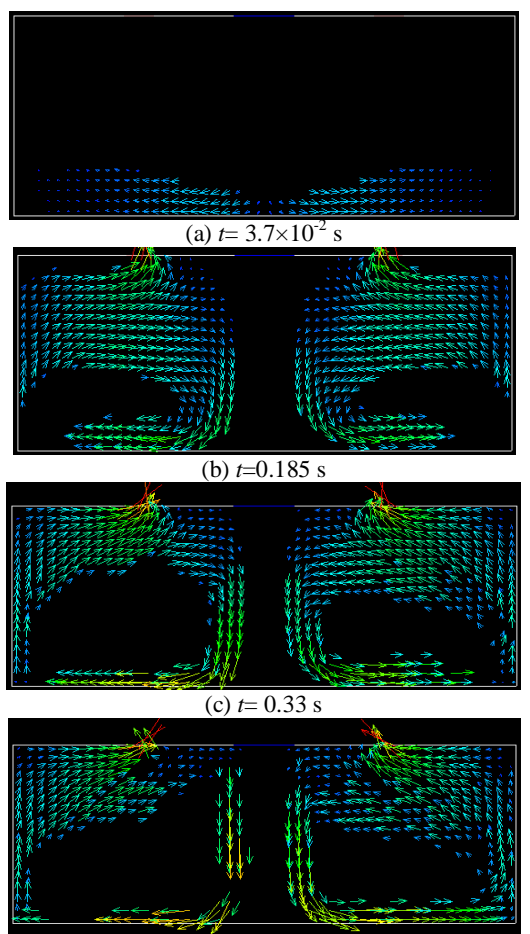

(d) $t=0.481 \mathrm{~s}$

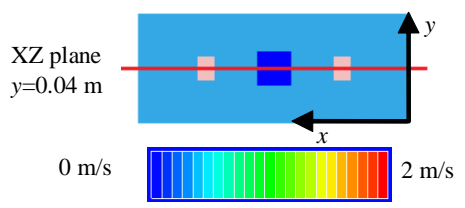

Fig. 4. Particle velocity vectors in plane $X Z$ from simulation (Case 1, y=0.04 m)

with the experimental data.

\subsection{Particle run-away rate}

To clarify the difference of particle movement in Cases 1 and 2 quantitatively, the particle run-out rate, $R_{\text {out }}$, which denotes the percentage of particle run out from

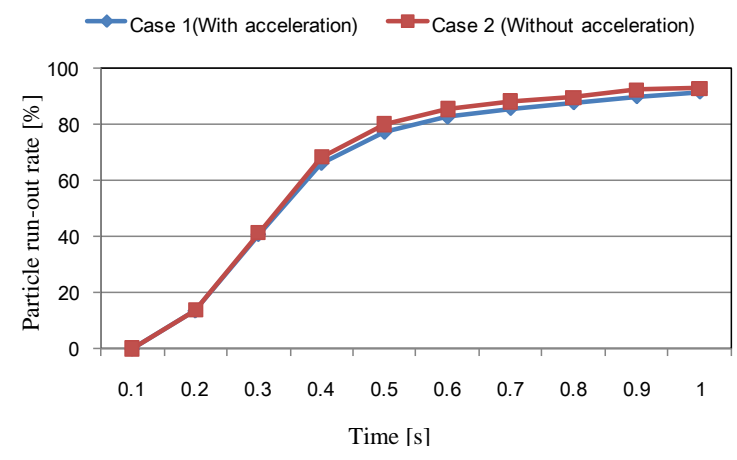


Fig. 6. Particle run-away rate under different acceleration condition

the outlet at elapsing time $t$, can be calculated in the simulation as follows:

$$
R_{\text {out }}=\frac{\sum_{1}^{n} N_{\text {out }}(n t)}{N_{p}} \times 100 \%
$$

where $N_{p}$ is the initial loading particle number; and $\mathrm{N}_{\text {out }}$ (nt) denotes the number of particles that leave from the outlet during the time interval $\Delta t$, where $n=1,2,3 \ldots$, and $\Delta t=0.1 \mathrm{~s}$. Figure 6 shows the calculated results for Cases 1 and 2 .

Figure 6 clarifies that, initially, particles run out at almost the same speed in the cases with or without acceleration. However, after $t=0.3 \mathrm{~s}$, the particle runaway rate under the acceleration condition becomes lower than that without acceleration. Eventually, the particle run- away rate stops at approximately $93 \%$ in the case without acceleration, and approximately $91 \%$ in the case with acceleration, which indicates that the acceleration adversely affects the particle run-away rate.

\section{CONCLUSION}

A numerical simulation for particle dispersion behavior in the cabin under acceleration was performed by combining the DEM and CFD in a three-dimensional domain. Distribution images of the particle concentration and velocity distribution were obtained from the simulation and compared with the experiment using the laser measurement method and PIV. The results are summarized as follows:

Two circulation particle flows with opposite circulating direction were generated under both with and without acceleration conditions. Except that a relatively high concentration areas were generated near the right wall of the calculation domain in the case with acceleration because of the relative acceleration between the calculation domain and the particles. In terms of the particle concentration distribution, the simulation results agree qualitatively with the laser measurement experiment, while the particle distribution is relatively dense, but little commonality can be found when the particle distribution becomes diluted due to the insufficient laser measurement point. The PIV measurement of the particle velocity distribution also shows a similar trend as the simulation result. All phenomena from the simulation are observed in the PIV measurement. The vortex is observed after the supplied air hits the stagnation point. The acceleration affects the particle motion and causes a relative high number of particles in the right vortex. Furthermore, the particle run- away rate under acceleration was initially the same as the condition without acceleration, but subsequently becomes lower which shows an adverse effect of the acceleration on the particle run- away rate.

\section{ACKNOWLEDGEMENTS}

The present study was supported in part by the National Natural Science Foundation of China (Grant No: 51275409) and the Science and Technology Program of
Shaanxi, China (Grant No: 2012JQ7009). Additional support was provided by the R-flow Corporation Ltd., Japan.

\section{REFERENCES}

Asgharzadeh H., Firoozabadi B. and Afshin H. (2012), Experimental and Numerical Simulation of the Effect of Particles on Flow Structures in Secondary Sedimentation Tanks, Journal of Applied Fluid Mechanics, 5(2), 15-23.

Abdul Rahim R., Green R. G., Horbury N., Dickin F.J., Naylor B. D. and Pridmore T. P. (1996), Further development of a tomographic imaging system using optical fibre for pneumatic conveyors, Measurement Science and Technology 7(3), 419422.

Bec J. (2003), Fractal clustering of inertial particles in random flows, Physics of Fluids 15, L81.

Bariter H.H. and Swindell W. (1996), Radiological Imaging: The Theory of Image Formation, Detection, and Processing. Waltham, United States: Academic Press.

Choi H.M., Kurihara T., Monji H. and Matsui G. (2002), Measurement of particle/bubble motion and turbulence around it by hybrid PIV, Flow Measurement and Instrumentation 12, 421-428.

Cundall P.A. and Strack O.D.L. (1979), A discrete numerical model for granular assemblies, Geotechnique 29, 47-65.

Ergun S. (1952), Fluid flow through packed columns, Chemical Engineering Progress 48, 89-94.

Fan W.Y., Ma Y.G., Li X.L. and Li H.Z. (2009), Study on the flow field around two parallel moving bubbles and Interaction Between Bubbles Rising in CMC Solutions by PIV, Chinese Journal of Chemical Engineering 17(6), 904-913.

Green R.G. and Abdul Rahim R.(1998), Concentration profiles in a gravity chute conveyor by optical tomography measurement, Powder Technology 95, 49-54.

Haller G., Sapsis T. (2008), Where do inertial particles go in fluid flows, Physica D: Nonlinear Phenomena 237, 573-520.

Kawaguchi T., Sakamoto M., Tanaka T. and Tsuji Y. (2000), Quasi-three-dimensional numerical simulation of spouted beds in cylinder, Powder Technology 109, 3-12.

Larsson I.A. S., Lindmark E. M., Lundström T. S., Marjavaara D. and Töyrä S. (2012), Visualization of Merging Flow by Usage of PIV and CFD with Application to Grate-Kiln Induration Machines, Journal of Applied Fluid Mechanics, 5(4), 81-89.

Michael O., Stephan G. and Frank B. (2009), EulerLagrange/DEM simulation of wood gasification in 
a bubbling fluidized bed reactor, Particuology 7 , 307-316.

Raffel M., Willert C.E., Wereley S.T. and Kompenhans J.(2007), Particle Image Velocimetry - A practical guide. Berlin, Germany: Springer-Verlag.

Shinbrot T., Alvarez M.M., Zalc J.M. and Muzzio F.J. (2001), Attraction of minute particles to invariant regions of volume preserving flows by transients, Physical Review Letter 86, 1207-1210.

Schmale D.G., Shah A.S. and Bergstro G.C. (2005), Spatial patterns of viable spore deposition of gibberella zeae in wheat fields, Phytopathology 95 , 472-479.

Shaw R.A. (2003), Particle-turbulence interactions in atmospheric clouds, Annual Review of Fluid Mechanics 35, 183-227.

Tirumkudulu M., Tripathi A. and Acrivos A. (1999), Particle segregation in monodisperse sheared suspensions, Physics of Fluids 11, 507-509.

Tsuji Y., Tanaka T. and Ishida T. (1992), Langrangian numerical simulation of plug flow of cohesionless particles in horizontal pipe, Powder Technology 71, 239-250.

Tsuji Y., Kawaguchi T. and Tanaka T. (1993), Discrete particle simulation of two-dimensional fluidized bed, Powder Technology 77, 79-87.
Wen C.Y. and Yu Y.H. (1966), Mechanics of fluidization, Chemical Engineering Progress Symposium Series 62, 100-111.

Wang X.F., Jin B.S., Xiong Y.Q. and Zhong W.Q. (2008), Flow Behaviors of Gas-Solid Injector by 3D Simulation with Kinetic Theory of Granular Flow, Chinese Journal of Chemical Engineering 16(6), 823-831.

Xu B.H., Yu A.B. (1997), Numerical simulation of the gas-solid flow in a fluidized bed by combining discrete particle method with computational fluid dynamics, Chemical Engineering Science 52, 2785-2809.

Zhao T. and Takei M. (2010), ECT measurement and CFD-DEM simulation of particle distribution in a down-flow fluidized bed, Flow Measurement and Instrumentation 21, 212-218.

Zhao T., Liu K., Cui Y.H. and Takei M. (2010), Threedimensional simulation of particle distribution in downer using CFD-DEM and comparison with ECT experiments, Advanced Powder Technology 21(6), 630-640.

Zheng Y., Liu Q., Li Y. and Gindy N. (2006), Investigation on concentration distribution and mass flow rate measurement for gravity chute conveyor by optical tomography system, Measurement 39, 643-654. 